\title{
Photoproduction of $\eta$ mesons on protons in the resonance region: The background problem and the third $S_{11}$ resonance.
}

\author{
V.A. Tryasuchev
}

October 29, 2018

\begin{abstract}
We have constructed an isobar model for the $\eta$-photoproduction on the proton in the energy region up to the photon lab energy $K_{0}=3 \mathrm{GeV}$. The data base involved into the fitting procedure includes precise results for the cross section and for the $T$-asymmetry of the process $\gamma p \rightarrow \eta p$ near threshold obtained at MAMI and ELSA as well as recent results for the $\Sigma$-asymmetry and for the angular distribution measured at higher energies in Grenoble and also more recent measurements performed at JLab for the photon energies up to 2 GeV. The model includes twelve nucleon resonances: $S_{11}(1535), S_{11}(1650), S_{11}(1825), P_{11}(1440), P_{13}(1720), D_{13}(1520), D_{15}(1675)$, $F_{15}(1680), F_{17}(1990), G_{17}(2190), G_{19}(2250), H_{19}(2220)$, and the background consisting of the nucleon pole term and the vector meson exchange in the $t$-channel. To explain the observed energy dependence of the integrated cross section, two $s$-wave resonances, $S_{11}(1650)$ and $S_{11}(1825)$, have to be taken into account along with the dominating $S_{11}(1535)$. The integrated cross section as well as the angular distribution and $\Sigma$ asymmetry predicted by the model are in good agreement with the data. Above the photon energy $K_{0}=2 \mathrm{GeV}$, the calculated cross section exhibits an appreciable dependence on the $\rho$ - and $\omega$-meson contribution, whose coupling with nucleons is not well defined. Several versions of extending the model to higher energies are considered.
\end{abstract}

\section{Introduction}

Information on the electromagnetic properties of the nucleon resonances are necessary for constructing the dynamical quark model of baryons. Photoproduction of pions on nucleons and nuclei remains a primary source of such information. On the other hand, because of a variety of $N^{*}$ and $\Delta$ resonances excited in the pion production, it is rather difficult to extract unambiguously their properties from the measured observables. Contrary to the pions, the $\eta$-photoproduction

$$
\gamma+p \rightarrow \eta+p
$$

involves only the $N^{*}$ resonances with isospin $I=\frac{1}{2}$, which make it possible to separate their contribution from the nearby $\Delta$ resonances $\left(I=\frac{3}{2}\right)$, appearing in the reactions with pions. It should also be noted that in spite of the common resemblance of process (11) to the pion photoproduction on a nucleon, there is a principle difference: Whereas the $\pi$-meson consists of the $u$ - and $d$-quarks, the $\eta$-meson includes also the strange quark-antiquark pair.

Apart from obtaining the information on the electromagnetic properties of the nucleon resonances, sophisticated models for process (11) are also needed to study the $\eta$-photoproduction on nuclei, which in turn is necessary for an investigation of the $\eta$-nuclear interaction. The modern $\eta$ photoproduction analysis can also be useful for estimating various background reactions in experiments with $\eta$ mesons.

\section{Description of a model}

In the center-of-mass system, the amplitude $F$ of photoproduction of a pseudoscalar meson on a nucleon reads [1]

$$
F=i F_{1} \vec{\sigma} \cdot \vec{\varepsilon}+F_{2} \vec{\sigma} \cdot \hat{q}(\hat{k} \times \vec{\varepsilon})+i F_{3} \vec{\sigma} \cdot \hat{k} \hat{q} \cdot \vec{\varepsilon}+i F_{4} \vec{\sigma} \cdot \hat{q} \hat{q} \cdot \vec{\varepsilon},
$$

where $\vec{\sigma}$ are Pauli matrices, representing the nucleon spin operator; $\hat{k}=\vec{k} / k$ and $\hat{q}=\vec{q} / q$ with $\vec{k}$ and $\vec{q}$ being the photon and $\eta$ c.m. momenta, and the unit vector $\vec{\varepsilon}$ determines the photon polarization. The four values $F_{i}$ are functions of 
the total energy $W$ and the meson c.m. angle $\theta$. In the angular momentum representation, $F$ 's are expressed in terms of electric $E_{l \pm}$ and magnetic $M_{l \pm}$ multipole amplitudes [1, 2]

$$
\begin{aligned}
& F_{1}=\sum_{l=0}^{\infty}\left[l M_{l^{+}}+E_{l^{+}}\right] P_{l+1}^{\prime}(x)+\sum_{l=0}^{\infty}\left[(l+1) M_{l^{-}}+E_{l^{-}}\right] P_{l-1}^{\prime}(x), \\
& F_{2}=\sum_{l=1}^{\infty}\left[(l+1) M_{l^{+}}+l M_{l^{-}}\right] P_{l}^{\prime}(x) \\
& F_{3}=\sum_{l=1}^{\infty}\left[-M_{l^{+}}+E_{l^{+}}\right] P_{l+1}^{\prime \prime}(x)+\sum_{l=3}^{\infty}\left[M_{l^{-}}+E_{l^{-}}\right] P_{l-1}^{\prime \prime}(x), \\
& F_{4}=\sum_{l=1}^{\infty}\left[M_{l^{+}}-E_{l^{+}}-M_{l^{-}}-E_{l^{-}}\right] P_{l}^{\prime \prime}(x),
\end{aligned}
$$

where $P_{l}(x)=P_{l}(\cos \theta)$ are Legendre polynomials. The observables of process (11) can be expressed in terms of the amplitudes $F_{i}$ as follows. Differential cross section:

$$
\begin{aligned}
\frac{d \sigma}{d \Omega} & =\frac{q}{k}\left\{\left|F_{1}\right|^{2}+\left|F_{2}\right|^{2}-2 \mathcal{R} e\left(F_{1} F_{2}^{*}\right) \cos \theta\right. \\
& \left.+\frac{1}{2}\left[\left|F_{3}\right|^{2}+\left|F_{4}\right|^{2}+2 \mathcal{R} e\left(F_{1} F_{4}^{*}\right)+2 \mathcal{R} e\left(F_{2} F_{3}^{*}\right)+2 \mathcal{R} e\left(F_{3} F_{4}^{*}\right) \cos \theta\right] \sin ^{2} \theta\right\} .
\end{aligned}
$$

$\Sigma$-asymmetry due to the linear photon beam polarization parallel and perpendicular to the reaction plane:

$$
\Sigma \frac{d \sigma}{d \Omega}=-\frac{q}{k} \frac{\sin ^{2} \theta}{2}\left[\left|F_{3}\right|^{2}+\left|F_{4}\right|^{2}+2 \mathcal{R} e\left(F_{2} F_{3}^{*}+F_{1} F_{4}^{*}+F_{3} F_{4}^{*} \cos \theta\right)\right] .
$$

$T$-asymmetry due to the initial nucleon polarization parallel and perpendicular to the reaction plane:

$$
T \frac{d \sigma}{d \Omega}=\frac{q}{k} \sin \theta \mathcal{I} m\left[F_{1} F_{3}^{*}-F_{2} F_{4}^{*}+\left(F_{1} F_{4}^{*}-F_{2} F_{3}^{*}\right) \cos \theta-F_{3} F_{4}^{*} \sin ^{2} \theta\right] .
$$

$P$-asymmetry due to the final nucleon polarization:

$$
P \frac{d \sigma}{d \Omega}=\frac{q}{k} \sin \theta \mathcal{I} m\left[F_{2} F_{4}^{*}-2 F_{1} F_{2}^{*}-F_{1} F_{3}^{*}+\left(F_{2} F_{3}^{*}-F_{1} F_{4}^{*}\right) \cos \theta+F_{3} F_{4}^{*} \sin ^{2} \theta\right] .
$$

Nowadays there are rigorous models for process (1) which describe very accurately the cross section in the region near threshold [3, 4, 5, 6, 7. At higher photon energy, due to relatively large $\eta$ mass, the higher resonances corresponding to the states with larger angular momentum are expected to be intensively involved into the production mechanism. Such states can simply be included into the model by using the Breit-Wigner ansatz for the $s$-channel part of the corresponding partial amplitudes. Therefore, the resonance multipoles were taken in the form [8]

$$
E_{l^{ \pm}}=\frac{e^{i \Phi_{r}}\left(\Gamma_{\gamma p}^{E} \Gamma_{\eta p}\right)^{1 / 2}}{2[k q j(j+1)]^{1 / 2}\left(W_{r}-W-\frac{i}{2} \Gamma\right)}
$$

and the analogical expression for the magnetic amplitudes $M_{l \pm}$. Here $l$ stands for the meson angular momentum, and $j=l \pm 1$ for $E_{l \pm}$ and $j=l$ for $M_{l \pm}$ respectively. The total $\eta N$ angular momentum is $J=l \pm \frac{1}{2}$. The energy $W_{r}$ is the total energy of the $\eta N$ system at the resonance position. Energy dependence of the total width $\Gamma$ was chosen according to the prescription given in Ref. 8

$$
\Gamma=\sum_{\alpha} \frac{p \nu_{l}(p R)}{p_{r} \nu_{l}\left(p_{r} R\right)} \Gamma_{\alpha r},
$$

where $\Gamma_{\alpha r}$ is the partial decay width in the channel $\alpha$, and the momenta $p$ and $p_{r}$ are the meson c.m. momenta corresponding to the energies $W$ and $W_{r}$, respectively. The function $\nu_{l}(x)$ stands for penetration factors, associated 
with the partial wave $l$. For $l \leq 3$, the needed expressions for $\nu_{l}(x)$ are given in 2 . Since for $l>3$ these factors are rarely presented in the literature we list here the expressions for $\nu_{l}(x)$ used in our model

$$
\begin{aligned}
& \nu_{0}(x)=1 \\
& \nu_{1}(x)=\frac{x^{2}}{1+x^{2}}, \\
& \nu_{2}(x)=\frac{x^{4}}{9+3 x^{2}+x^{4}}, \\
& \nu_{3}(x)=\frac{x^{6}}{225+45 x^{2}+6 x^{4}+x^{6}}, \\
& \nu_{4}(x)=\frac{x^{8}}{11025+1575 x^{2}+135 x^{4}+10 x^{6}+x^{8}}, \\
& \nu_{5}(x)=\frac{x^{10}}{893025+99225 x^{2}+6300 x^{4}+315 x^{6}+15 x^{8}+x^{10}} .
\end{aligned}
$$

In Eq. (9) the effective radius of $\eta N$-interaction $R$, was taken to be equal to $1 \mathrm{fm}$ and was not varied in the fitting procedure. For all resonances, but $S_{11}(1535)$, the sum in (9) was restricted to the pion channel. For the $S_{11}(1535)$ resonance which quite intensively decays into the $\eta N$ channel the energy dependent width was taken in the form

$$
\Gamma=\left(0.5 \frac{q}{q_{r}}+0.4 \frac{p}{p_{r}}+0.1\right) \Gamma_{r},
$$

where $q$ and $p$ are c.m. momenta of $\eta$ and $\pi$ mesons, respectively, and the corresponding values $\Gamma_{r}, q_{r}$, and $p_{r}$ are calculated at the resonance position $W=W_{r}$. [8]

The electromagnetic $\Gamma_{\gamma p}^{E, M}$ and strong $\Gamma_{\eta p}$ vertices of the resonance amplitudes (8) were parametrized in the form

$$
\left(\Gamma_{\gamma p}^{E, M} \Gamma_{\eta p}\right)^{1 / 2}=\left\{\left[2 k R \nu_{n}(k R)\right]\left[2 q R \nu_{l}(q R)\right]\right\}^{1 / 2} \gamma^{E, M}
$$

where $n=l$, except for the multipole amplitudes $E_{l^{-}}$, for which $n=l-2$. The values $\gamma^{E, M}$ were treated as free parameters together with the main resonance characteristics $W_{r}$ and $\Gamma_{r}$.

Apart from the resonance terms, smooth background contributions consisting of the nucleon pole terms in the $s$ and $u$-channel and exchange of $\rho$ - and $\omega$-mesons in the $t$-channel were included into the model. The nucleon pole amplitudes read $\underline{5}$

$$
\begin{aligned}
& F_{1}=e g_{\eta N N} \frac{W+m}{8 \pi W} \sqrt{\frac{E_{f}+m}{E_{i}+m}} k\left[e_{N}+\mu_{N}\right]\left(\frac{1}{s-m^{2}}+\frac{1}{u-m^{2}}\right), \\
& F_{2}=-e g_{\eta N N} \frac{W-m}{8 \pi W} \sqrt{\frac{E_{i}+m}{E_{f}+m}} q\left[e_{N}+\mu_{N}\right]\left(\frac{1}{s-m^{2}}+\frac{1}{u-m^{2}}\right), \\
& F_{3}=2 e g_{\eta N N} \frac{W+m}{8 \pi W} \sqrt{\frac{E_{f}+m}{E_{i}+m}} q k\left[\frac{e_{N}}{W+m}+\frac{\mu_{N}}{2 m}\right] \frac{1}{u-m^{2}}, \\
& F_{4}=-2 e g_{\eta N N} \frac{W-m}{8 \pi W} \sqrt{\frac{E_{i}+m}{E_{f}+m}} q^{2}\left[\frac{e_{N}}{W-m}-\frac{\mu_{N}}{2 m}\right] \frac{1}{u-m^{2}},
\end{aligned}
$$

where $m$ is the nucleon mass; $g_{\eta N N}$ is the constant of the $\eta N N$ pseudoscalar coupling, and $e_{N}$ and $\mu_{N}$ stand for the charge $\left(e_{p}=1, e_{n}=0\right)$ and the anomalous magnetic moment $\left(\mu_{p}=1.79, \mu_{n}=-1.91\right)$ of the nucleon; $e^{2} / 4 \pi$ is the fine-structure constant.

For the vector meson contribution one obtaines [5]

$$
\begin{aligned}
& F_{1}=e \lambda_{V} \frac{W+m}{8 \pi W m_{\eta}} \sqrt{\frac{E_{f}+m}{E_{i}+m}} k\left[\frac{G_{V N N}^{t}}{2 m}-G_{V N N}^{v}\left(W-m+\frac{t-m_{\eta}^{2}}{2(W-m)}\right)\right] \frac{1}{t-m_{V}^{2}}, \\
& F_{2}=-e \lambda_{V} \frac{W-m}{8 \pi W m_{\eta}} \sqrt{\frac{E_{i}+m}{E_{f}+m}} q\left[\frac{G_{V N N}^{t}}{2 m}+G_{V N N}^{v}\left(W+m+\frac{t-m_{\eta}^{2}}{2(W+m)}\right)\right] \frac{1}{t-m_{V}^{2}},
\end{aligned}
$$




$$
\begin{aligned}
& F_{3}=-e \lambda_{V} \frac{W+m}{8 \pi W m_{\eta}} \sqrt{\frac{E_{f}+m}{E_{i}+m}} k q\left[\frac{G_{V N N}^{t}}{2 m}(W-m)-G_{V N N}^{v}\right] \frac{1}{t-m_{V}^{2}}, \\
& F_{4}=e \lambda_{V} \frac{W-m}{8 \pi W m_{\eta}} \sqrt{\frac{E_{i}+m}{E_{f}+m}} q^{2}\left[\frac{G_{V N N}^{t}}{2 m}(W+m)+G_{V N N}^{v}\right] \frac{1}{t-m_{V}^{2}} .
\end{aligned}
$$

Here $\lambda_{V}$ is the $\gamma \eta V$ coupling constant and the values

$$
G_{V N N}^{v, t}=g_{V N N}^{v, t} G^{V}(t)
$$

are the constants determining vector and tensor $V N N$ coupling.

Our main task was to develop the model which could provide a good description of the low energy data for $\gamma p \rightarrow \eta p$ and at the same time has a "right" asymptotic behavior at higher photon energies $\left(K_{0}>2 \mathrm{GeV}\right)$.

\section{Discussion of the results}

Varying the parameters of the resonances listed in Table 1 within the ranges recommended in Ref. [10] we obtain a good description of the data for $\gamma p \rightarrow \eta p$. At the beginning also the relative phases $\Phi_{r}$ (see Ref. [11]) were used in the fitting procedure, but after the eleven resonances and the background terms were included into the model, we were able to abandon these phase factors at all 12. The resulting set of parameters listed in Table 10 [13 was obtained by adjusting the model containing all eleven resonances and the background to the low energy data $\left(K_{0}<1.1 \mathrm{GeV}\right)$ presented in Ref. 14, 15, 16, 17] as well as to the old experimental results for $K_{0}>1.2 \mathrm{GeV}$ obtained in [18, 19].

As was repeatedly noted in the previous work, the dominant contribution to the cross section of $\gamma p \rightarrow \eta p$ arises from the $S_{11}(1535)$ resonance, which is strongly coupled to the $\eta N$ channel. However inclusion of this resonance alone does not reproduce energy dependence exhibited by the measured total cross section. Only including $S_{11}(1650)$, and damping by this means too a large contribution of $S_{11}(1535)$ resonance at the energies above $0.8 \mathrm{GeV}$, we are able to bring the theory into agreement with the data which were obtained in two experiments in different energy regions (see Fig.(1). Also the resonance $P_{13}(1720)$ provides within our model an essential contribution to the $\eta$ photoproduction amplitude.

Using the parameters of $S_{11}(1535)$ from Table 1 one obtains

$$
\Gamma_{\gamma} / \Gamma_{r} \approx 0.345 \%,
$$

where $\Gamma_{\gamma}$ denotes the total radiative width of the resonance. This result agrees with the maximal value of this ratio, recommended in Ref. [10]. The same result can be presented in the form

$$
\Gamma_{\eta p} \Gamma_{\gamma} / \Gamma_{r} \approx 0.27 \mathrm{MeV} .
$$

Then, taking $\Gamma_{\eta p} / \Gamma_{r}=0.50$, one obtains for the invariant helicity amplitude $A_{1 / 2}=0.110 \mathrm{GeV}^{-1 / 2}$. And conversely, taking $A_{1 / 2}=0.090 \mathrm{GeV}^{-1 / 2}$, as is recommended in [10, one finds the ratio of the widths $\Gamma_{\eta p} / \Gamma_{r}=0.74$. This result points to the fact that the role of the $S_{11}(1535)$ resonance in the process $\gamma N \rightarrow \eta N$ appears to be greater than the one prescribed by the PDG analysis [10]. At the same time, the value of the $S_{11}(1535)$ photoexcitation amplitude $A_{1 / 2}$ is in good agreement with $A_{1 / 2}=0.118 \mathrm{GeV}^{-1 / 2}$, obtained in Ref. 9]. As for the resonance $S_{11}(1650)$, if one takes $\Gamma_{\eta p} / \Gamma_{r}=0.1$ then $\Gamma_{\gamma} / \Gamma_{r} \approx 0.38 \%$ and $A_{1 / 2}=0.098 \mathrm{GeV}^{-1 / 2}$. The latter value is about two times larger then the upper limit, which can be found in Ref. 10. For the resonance $P_{13}(1720)$ the constants $\gamma^{E, M}$ obtained by fitting the data lead to the large absolute significance of the values $\xi_{\lambda}$ (see Table 1). More detailed information about the electromagnetic vertices of $N^{*}$ resonances can be obtained from the results presented in Table 1] if the corresponding ratios $\Gamma_{\eta p} / \Gamma_{r}$ are known.

Among the resonances with larger masses, only those marked in [10] with four asterisks were included into the fitting procedure, namely $F_{17}(1990), G_{17}(2190), G_{19}(2250)$, and $H_{19}(2220)$. As the direct calculations shows, insertion of these resonances influences mostly the $\Sigma$ asymmetry, while the differential and total cross sections in the region $K_{0}<1.2 \mathrm{GeV}$ are less affected. The most important contribution to $\gamma p \rightarrow \eta p$ comes from the $G_{19}(2250)$ resonance.

The calculated angular distributions $d \sigma / d \Omega$ are in excellent agreement with the experimental results reported by MAMI (Mainz) and GRAAL (Grenoble) up to the photon energy $K_{0} \approx 950 \mathrm{MeV}$. They were discussed in our previous work [1] and are not presented here. With increasing energy the theoretical distributions $d \sigma / d \Omega$ exhibit pronounced decrease at forward angles (see also Ref. [9]) which was not observed in the measurements [15]. Some results around 
the energy $K_{0}=1 \mathrm{GeV}$, which were obtained in the present as well as in the previous work [1] are compared with the data in Fig.2] It must be emphasized that the model including eleven [12] resonances agrees with the experimental results at these energies as good as the low energy model [1] with six resonances. Furthermore, we would like to note that the maxima in $\Sigma(\theta)$ for $K_{0}>950 \mathrm{MeV}$ appear at the same angles $\left(\theta \approx 50^{\circ}\right)$ as does the maxima of $d \sigma / d \Omega$ at the corresponding energies. This is the reason why the experimental values of $\Sigma(\theta)$ increase strongly at the energy around $K_{0}=1 \mathrm{GeV}$ (see Fig.(2).

The target asymmetry $T(\theta)$ (6) predicted by the model is positive. Furthermore, it depends almost not at all on the born (nucleon pole) term, as well as on the form factors in the $V N N$ vertices and agrees qualitatively with the Bonn results [17, as is shown in Fig. [3] In the same figure we also demonstrate the sensitivity of $T(\theta)$ to the contribution of the $P_{13}(1720)$ and $D_{15}(1675)$ resonances. It must also be noted, that the model of [11 (the result is shown by the doted curve) was in disagreement with the experimental results. At the same time, we could not describe the experimental data of work [17] on the target asymmetry of process (1) for the photon lab energy below $750 \mathrm{MeV}$ by using the resonances from table 1 .

Finally, the polarization of the final protons $P(\theta)$ (7) is in strong disagreement with the experimental data obtained 30 years ago 20 .

\section{Investigation of the background}

If the background contribution is not included, the integrated cross section decreases rapidly to zero. Therefore, a pure resonance model for $\gamma p \rightarrow \eta p$ seems to be incompatible with a real production dynamics. As was noted above, the background contains the nucleon pole term in $s$ and $u$ channels as well as $\rho$ and $\omega$ exchanges in $t$ channel. The nucleon pole term is defined by the $\eta N N$ coupling constant. Its contribution appears to be insignificant for all reasonable values of $g_{\eta N N}$, therefore we take $g_{\eta N N}^{2} / 4 \pi=0.4$ from Ref. [5] [7, 9].

For pointlike $V N N$ vertices with $G^{V}(t)=1$ the $\rho$ and $\omega$ contributions rise rapidly with increasing photon energy so that for the photon energies above $K_{0}=1.5 \mathrm{GeV}$ the photoproduction amplitude is almost totally determined by the vector meson exchange, and the cross section is anomalously enhanced in the region close to $K_{0}=3 \mathrm{GeV}$. Taking into account a finite range of the $V N N$ coupling $\left(G^{V}(t) \rightarrow 0\right.$ if $\left.|t| \rightarrow \infty\right)$ leads to visible damping of the cross section(see Fig.(4). However,its value depends strongly on the choice of the $V N N$ form factors. In the present work we have considered two types of form factors

$$
G^{V}(t)=\left(\frac{\Lambda_{V}^{2}-m_{V}^{2}}{\Lambda_{V}^{2}-t}\right)^{n}
$$

with $n=1$ (monopole type [6]) and $n=2$ (dipole type [5, 9, 21]) with different cut off parameters $\Lambda_{V}$. Whereas the constants $\lambda_{V}$ in Eq. (14) are quite well known from the radiation decays $V \rightarrow \gamma \eta$, (see, e.g., [9]), the hadronic constants $g_{V N N}^{v}$ and $g_{V N N}^{t}$ are determined poorly for both $\rho$ and $\omega$ mesons, and are usually treated as free parameters in phenomenological models. Several sets of these constants, used in the previous work, are listed in Table 2 Appreciable difference between the total cross section obtained with these sets appears only above the energy $K_{0}=1.9 \mathrm{GeV}$ (see Fig.(4). It must be kept in mind that all but the latter sets given in Table 2 were used for the description of $\gamma N \rightarrow \eta N$ observations only in the low-energy region $\left(K_{0}<1.1 \mathrm{GeV}\right)$.

\section{$5 \quad$ Third $S_{11}$ resonance in $\gamma p \rightarrow \eta p$}

In the previous work [12] we have extended the model to higher energies $\left(K_{0}>1.2 \mathrm{GeV}\right)$ using the old database from [18] 19, since the experimental results obtained at JLab 22] for $K_{0}=0.75-1.95 \mathrm{GeV}$ were published later. The data [22] agree with those published in [15] at $K_{0}=0.75-1.0 \mathrm{GeV}$ but above this energy region one notes a visible deviation (Fig 5). Our model with eleven resonances does not reproduce the results of [22]. The same deviation can also be observed by comparing the data with other analyzes, e.g [21]. In order to eliminate this disagreement we have introduced into the model, apart from two $S_{11}$ resonances with masses 1535 and $1650 \mathrm{MeV}$, the third $S_{11}$ resonance with the parameters given in Table 1 (marked by asterisk). Varying the ratio $\Gamma_{\eta p} / \Gamma_{r}$ in the region 0.5 to 0.01 one obtains for the invariant helicity amplitude of the third $S_{11}$ resonance the $A_{1 / 2}=0.021$ to $0.151 \mathrm{GeV}^{-1 / 2}$, appreciably smaller than the corresponding values of other two $s$-wave resonances. The effect of including the $S_{11}(1825)$ resonance into the model of Ref. [12 is shown in Fig. [6] The contribution vanishes totally at the ends of the interval $K_{0}=1.025-1.9 \mathrm{GeV}$, as is seen in Figs. 6a and 6d, which in turn guarantees that a quite good description of the 
data achieved in the low-energy region within the model with eleven resonances is not affected by insertion of the new resonance. There are appreciable differences between the differential cross sections at forward and backward $\eta$ angles predicted by various models $9,12,23,24$. Therefore to discriminate between the models, measurements of the $\eta$ angular distribution at small $\left(\theta<45^{\circ}\right)$ and large angles $\left(\theta>135^{\circ}\right)$ became important. The main distinctive property of the present model is, that it takes explicitly into account the contribution of higher resonances with large angular momenta, $F_{17}(1990), G_{17}(2190), G_{19}(2250), H_{19}(2220)$, which make the angular distribution of $\gamma N \rightarrow \eta N$ at $K_{0}>1.5 \mathrm{GeV}$ strongly anisotropic (Fig [6). In this connection we take with some caution the estimation of the total cross section made by the authors of Ref. [22] who has used only the values of $d \sigma / d \Omega$ in the interval $45^{0}<\theta<135^{0}$. As is noted above, such estimation must depend strongly on the model used for the extrapolation of the cross section into the region of small and large $\eta$ angles. The dependencies of the angular distributions of the $\eta$ mesons in process (1) for energy region $K_{0}>1.5 \mathrm{GeV}$ on contributions of higher-spin resonances are shown in Fig 7 The differential cross section form depends significantly on contributions of the resonances $F_{17}(1990), G_{17}(2190), G_{19}(2250)$. The influence of the contribution of the resonance $H_{19}(2220)$ on it is minor. In this energy region the calculations have shown that higher-spin resonances are responsible for the large positive $\Sigma$ beam asymmetry.

The third $s$-wave resonance discussed here is not heuristic. This resonance was predicted by quark models and was also observed in other processes [22, 23, 24, 25, 26]. Its parameters, needed to describe the data (see Table 1) are in surprisingly good agreement with those, extracted from the experimental results for the decay $J / \Psi \rightarrow p \bar{p} \eta$ 23] $\left(W_{r}=1800 \pm 40 \mathrm{MeV}, \Gamma_{r}=165_{-85}^{+165} \mathrm{MeV}\right)$, but do not agree with the properties of the third $S_{11}$ resonance predicted by the constituent quark model with broken $S U(6) \otimes O(3)$ symmetry [24] $\left(W_{r}=1776 \mathrm{MeV}, \Gamma_{r}=268\right.$ $\mathrm{MeV}$ ). While on the subject of the new $S_{11}$ resonances, we would like to note the work [25] where a unified analysis of the data for the scattering and photoproduction of pions in a large energy region was performed. The results of the cited work point to the existence of the third and the forth $S_{11}$ resonances with masses $M_{r}=1846 \pm 47 \mathrm{MeV}$ and $M_{r}=2113 \pm 70 \mathrm{MeV}$ respectively. However, the corresponding widths can not be defined with such a precision and their values were estimated to be more than $300 \mathrm{MeV}$. Although the authors of Ref. 21] do not introduce additional $s$-wave resonance for the description of the $\gamma p \rightarrow \eta p$ cross section, they have to insert it when explaining the the data for $\gamma p \rightarrow \eta^{\prime} p$. Finally, the third $S_{11}$ resonance is predicted also in the hypercentric constituent quark model [26] which gives $W_{r}=1861 \mathrm{MeV}$. In conclusion, the resonance $S_{11}$ which is needed for the description of the available data 22 . around $K_{0}=1350 \mathrm{MeV}$ has properties which agree quite well with the results obtained in the recent theoretical and experimental investigations.

\section{Perspectives of the model}

Using our model with twelve resonances (together with the third $S_{11}$ resonance), we have extended the calculation of the cross section for $\gamma p \rightarrow \eta p$ to higher photon energies. There are other theoretical predictions in the energy region above $K_{0}=2 \mathrm{GeV}$, from which we would like to note the Ref. 21] where the usual isobar model approach was supplemented by the Regge model. However, the results provided by this hybrid model in the region $K_{0}=2-4$ $\mathrm{GeV}$ are not in good agreement with the data. Extending our model to the energy $K_{0}=4 \mathrm{GeV}$ with background parameters from Ref. [9] we are faced again with unacceptable increase of the cross section above the energy $K_{0}=3.4$ $\mathrm{GeV}$ caused by anomalously large contribution of the vector meson exchange. Therefore, we have introduced the new set of $V N N$ parameters (fourth set in Table 21) and have obtained the result shown by the solid curve in Fig 8 Above the photon energy $K_{0}=1.5 \mathrm{GeV}$ a peripheral $\eta N$ interaction leading to the excitation of higher resonances $F_{17}(1990)$, $G_{17}(1990), G_{19}(2250)$, and $H_{19}(2200)$ becomes important. The latter are not well studied as yet, in particular since relatively large widths of these resonances (see Table 1) prevent an isolation in their individual contributions to the observables. New measurements in the high energy region $\left(K_{0}>2 \mathrm{GeV}\right)$ which could solve this problem as well as establish the properties of the fourth $S_{11}$ resonance [25] are very desirable.

I am grateful to A.I. Fix.

\section{References}

[1] G.F. Chew, M.L. Goldberger, F.E. Low and Y. Nambu, Phys. Rev. 106, 1345 (1957)

[2] J.M. Blatt and V. Weisskopf, Theoretical Nuclear Physics (N.Y., Wiley \& Sons, 1952)

[3] C. Bennhold and H. Tanabe, Nucl. Phys. A530, 62 (1991) 
[4] M. Benmerrouche and N.C. Mukhopadhyay, Phys. Rev. Lett. 67, 1070 (1991)

[5] L. Tiator, C. Bennhold, and S.S. Kamalov, Nucl. Phys. A580, 455 (1994)

[6] M. Benmerrouche, N.C. Mukhopadhyay, and J.F. Zhang, Phys. Rev. D51, 3237 (1995)

[7] A. Fix and H. Arenhövel, Nucl. Phys. A 620, 457 (1997)

[8] H.R. Hicks, S.E. Deans, D.T. Jacobs, and D.L.Montgomery, Phys. Rev. D7, 2614 (1973)

[9] W.-T. Chiang, S.N. Yang, L. Tiator, and D. Drechsel, Nucl. Phys. A700, 429 (2002)

[10] Particle Data Group, Eur. Phys. J. C15, 1 (2000)

[11] V.A. Tryasuchev, Russ. Phys. J. 44, 677 (2001); Phys. Atom. Nucl. 65, 1673 (2002)

[12] V.A. Tryasuchev, Russ. Phys. J. 46, 403 (2003)

[13] At this stage the third resonance $S_{11}(1825)$ marked by the asterisk in Table 1 was not included into the fitting procedure

[14] B. Krusche, J. Ahrens, G. Anton, et al., Phys. Rev. Lett. 74, 3736 (1995)

[15] F. Renard, M. Anghinolfi, O. Bartalini, et al., Phys. Lett. B528, 215 (2002)

[16] J. Ajaka, M. Anghinolfi, V. Bellini, et al., Phys. Rev. Lett. 81, 1797 (1998)

[17] A. Bock, et al., Phys. Rev. Lett. 81, 534 (1998)

[18] ABBHHM Collaboration, Phys. Rev. 175, 1669 (1968)

[19] G.A. Vartapetyan and S.E. Piliposyan, Sov. J. Nucl. Phys. 32, 804 (1980)

[20] C.A. Heusch, C.Y. Prescott, L.S. Rochester, et al., Phys. Rev. Lett. 25, 1381 (1970)

[21] W.-T. Chiang, S.N. Yang, L. Tiator, M. Vanderhaeghen, and D. Drechsel, Phys.Rev.C68, 045202 (2003)

[22] M. Dugger, B.G. Ritchie, J. Ball, et al., Phys. Rev. Lett. 89, 222002 (2002)

[23] J.Z. Bai, et al., Phys. Lett. B510, 75 (2001)

[24] B. Saghai, Z. Li, nucl-th/0202007

[25] G.-Y. Chen, S.S. Kamalov, S.Y. Yang, et al., Nucl. Phys.A723, 447 (2003)

[26] M.M. Giannini, E. Santopinto, A. Vassalo, Eur.Phys.J.A12 447 (2001) 
Table 1: Resonance parameters obtained by fitting the $\gamma p \rightarrow \eta p$ observations to the available data. The background was taken into account as in Ref. [21]. The values $\xi_{\lambda}=\sqrt{\frac{k m \Gamma_{\eta p}}{q W_{r}}} A_{\lambda}$ were introduced in Ref. [6].

\begin{tabular}{|c|c|c|c|c|c|c|}
\hline$N^{*}$ resonance & $W_{r}[\mathrm{MeV}]$ & $\Gamma_{r}[\mathrm{MeV}]$ & $\gamma^{E}[\mathrm{MeV}]$ & $\gamma^{M}[\mathrm{MeV}]$ & $\xi_{\frac{1}{2}}\left[10^{-1} \mathrm{GeV}^{-1}\right]$ & $\xi_{\frac{3}{2}}\left[10^{-1} \mathrm{GeV}^{-1}\right]$ \\
\hline$S_{11}(1535)$ & 1535 & 158 & 2.16 & - & 2.45 & - \\
$S_{11}(1650)$ & 1645 & 140 & -0.620 & - & 0.795 & - \\
$* S_{11}(1825)$ & 1825 & 160 & 0.275 & - & 0.308 & - \\
$P_{11}(1440)$ & 1440 & 350 & - & 0.250 & - & - \\
$P_{13}(1720)$ & 1722 & 145 & -0.105 & 0.430 & -0.462 & 0.642 \\
$D_{13}(1520)$ & 1520 & 120 & 0.200 & 0.330 & -0.044 & 0.117 \\
$D_{15}(1675)$ & 1673 & 150 & 0.115 & 0.260 & 0.261 & -0.156 \\
$F_{15}(1680)$ & 1680 & 130 & 0.045 & 0.050 & -0.0003 & 0.039 \\
$F_{17}(1990)$ & 1990 & 375 & -0.056 & -0.4130 & 0.153 & 0.150 \\
$G_{17}(2190)$ & 2190 & 400 & -0.175 & -0.240 & -0.018 & 0.126 \\
$G_{19}(2250)$ & 2250 & 450 & -0.322 & -0.600 & 0.253 & 0.105 \\
$H_{19}(2220)$ & 2220 & 450 & -0.120 & -0.700 & -0.078 & 0.111 \\
\hline
\end{tabular}

Table 2: Vector meson coupling constants used in the present work for the $t$-channel part of the $\gamma p \rightarrow \eta p$ process.

\begin{tabular}{|c|c|c|c|c|c|c|}
\hline Meson & Mass [MeV] & $\frac{\left(g_{V N N}^{v}\right)^{2}}{4 \pi}$ & $\frac{\left(g_{V N N}^{t}\right)^{2}}{4 \pi}$ & $\lambda_{V}$ & $\Lambda_{V}[\mathrm{GeV}]$ & Ref. \\
\hline$\rho$ & 770 & 0.50 & 18.6 & 0.89 & 1.80 & {$[\underline{5}$} \\
$\omega$ & 782 & 23.0 & 0 & 0.192 & 1.40 & \\
\hline$\rho$ & 770 & 0.55 & 20.5 & 1.06 & 1.089 & {$[\underline{6}$} \\
$\omega$ & 782 & 8.11 & 0.20 & 0.31 & 1.106 & \\
\hline$\rho$ & 768.5 & 0.458 & 17.5 & 0.81 & 1.3 & {$[9]$} \\
$\omega$ & 782.6 & 20.37 & 0 & 0.291 & 1.3 & \\
\hline$\rho$ & 768.5 & 0.458 & 1.09 & 0.81 & 1.0 & {$[21]$} \\
$\omega$ & 782.6 & 6.45 & 0 & 0.29 & 1.3 & \\
\hline
\end{tabular}




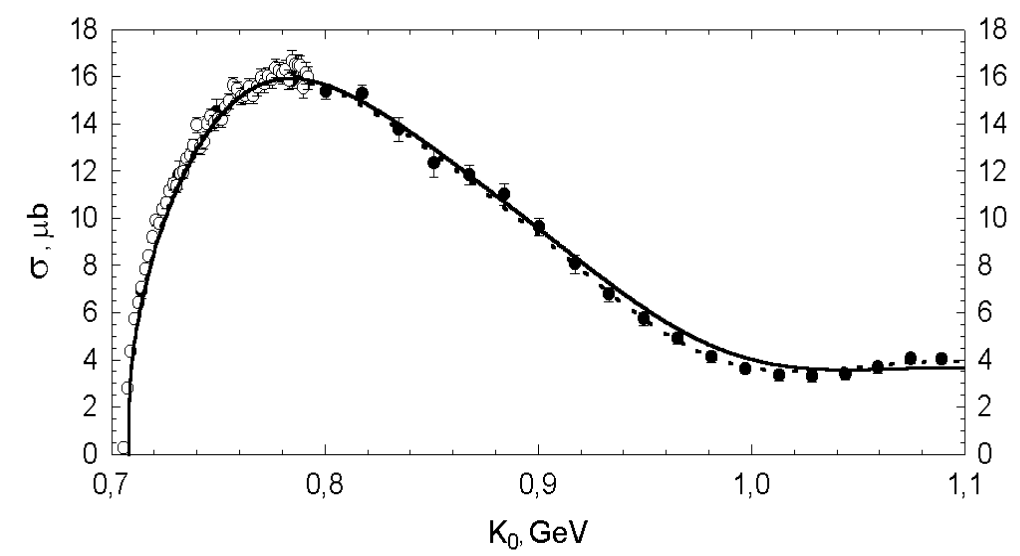

Figure 1: Integrated cross section for $\gamma p \rightarrow \eta p$ as a function of lab photon energy $K_{0}$. The prediction of the present model is shown by the solid curve. Doted curve is the result of the Ref. 11]. The data are from [14] (open circles) and [15] (filled circles).
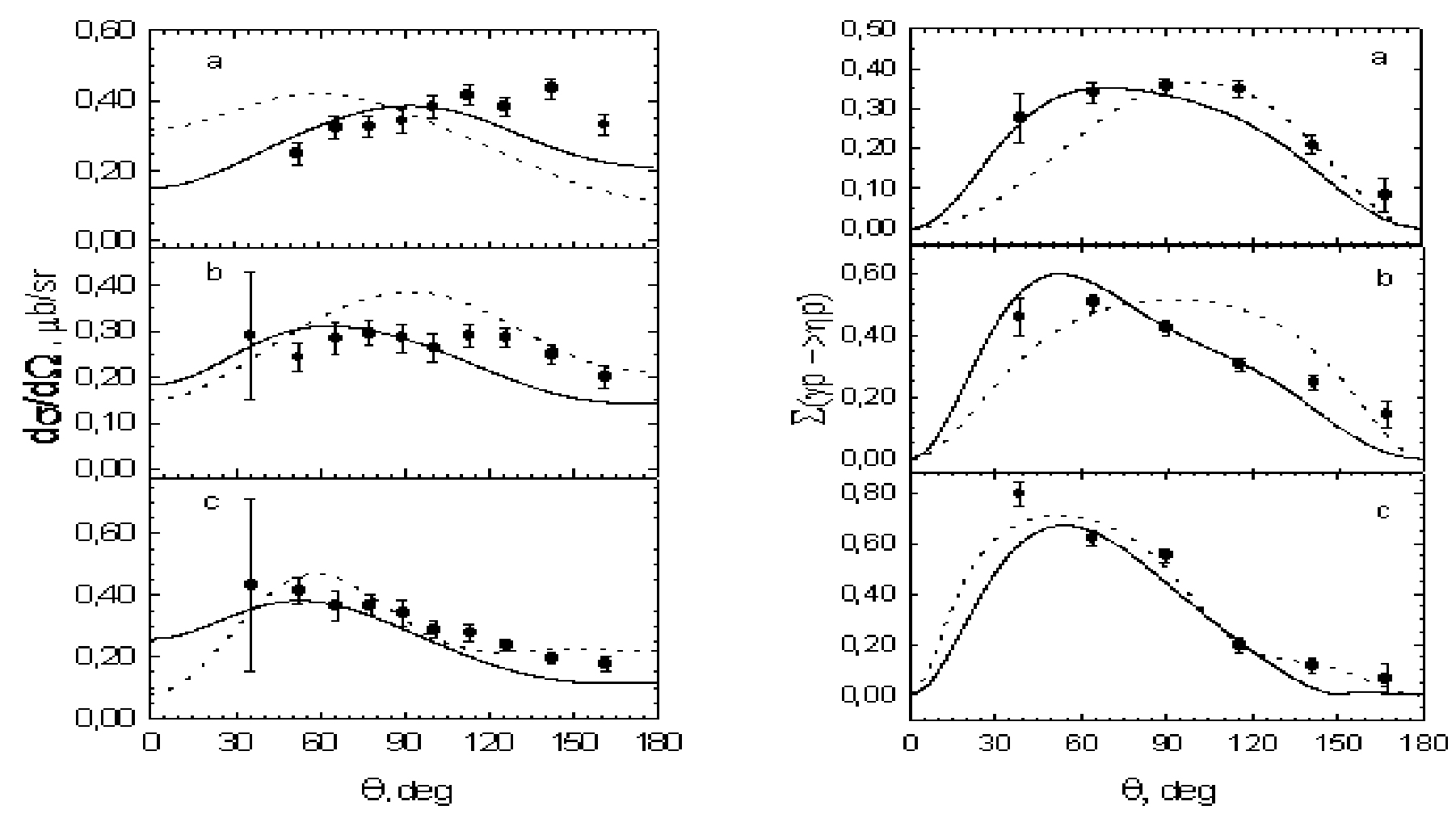

Figure 2: Left panel: differential cross section $d \sigma / d \Omega$ versus the $\eta$ c.m. emission angle. Shown are the calculations at three lab photon energies: $K_{0}=981 \mathrm{MeV}(\mathrm{a}), K_{0}=1029 \mathrm{MeV}(\mathrm{b})$, and $K_{0}=1075 \mathrm{MeV}$ (c). The data are taken from [15]. Solid curves are obtained using the model with eleven resonances with the parameters listed in Table 1 The doted curves are the results of the model [11. Right panel: The $\Sigma$ asymmetry calculated at $K_{0}=931 \mathrm{MeV}$ (a), $K_{0}=991 \mathrm{MeV}(\mathrm{b})$, and $K_{0}=1056 \mathrm{MeV}$ (c). The data are from Ref. 16. The meaning of the curves as in the left panel. 


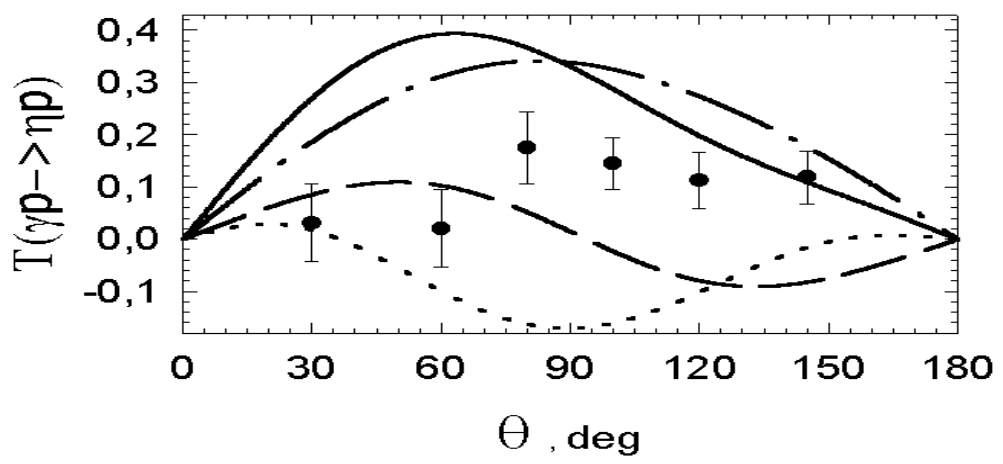

Figure 3: Target asymmetry for $\gamma p \rightarrow \eta p$ at $K_{0}=857 \mathrm{MeV}$ calculated in the lab system. The theoretical results are obtained using the present model with all resonances (solid curve), without $P_{13}(1720)$ (dashed curve), and without $D_{15}(1675)$ (dash-dotted curve). The dotted curve presents the result of Ref. [11]. The data are from [17.

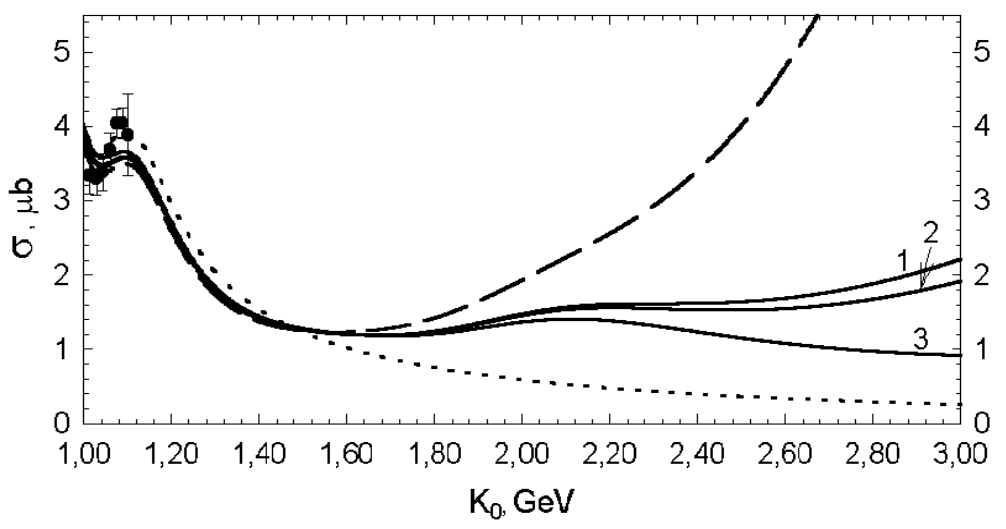

Figure 4: Integrated cross section for $\gamma p \rightarrow \eta p$ above the lab photon energy $K_{0}=1 \mathrm{GeV}$. The solid curves 1,2 , and 3 are calculated with vector meson exchange parameters from Refs. [5], 6] and [9], respectively (see Table 22). The dashed curve includes the vector meson exchange from Ref. [5] but with $G^{V}(t)=1$. The dotted curve is the result of the model [11. The data are taken from Ref. [15]. 


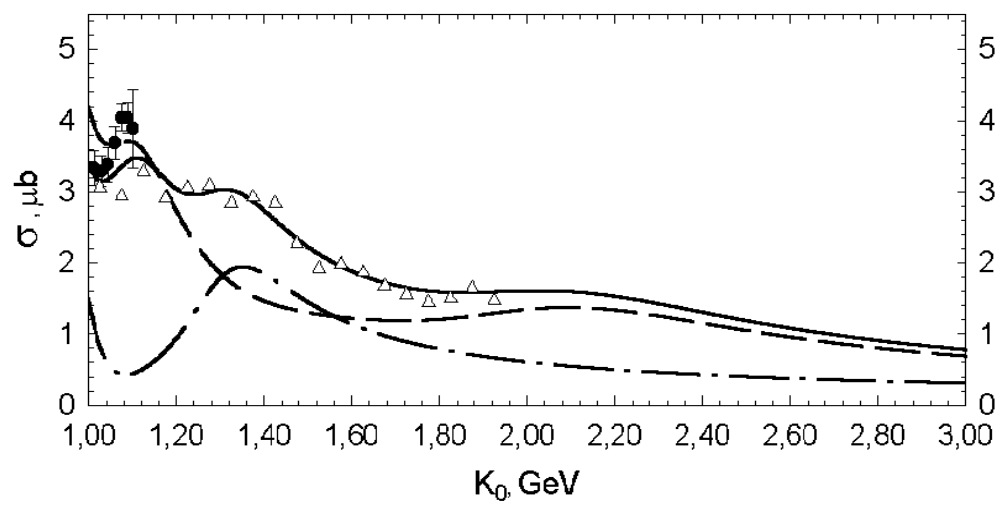

Figure 5: Integrated cross section for $\gamma p \rightarrow \eta p$. The dashed and solid curves are obtained using the model with eleven and twelve resonances, respectively, plus background. The dash-dotted curve includes only $s$-wave resonances from Table 1 plus background. The data are taken from [15] (circles) and [22] (triangles).

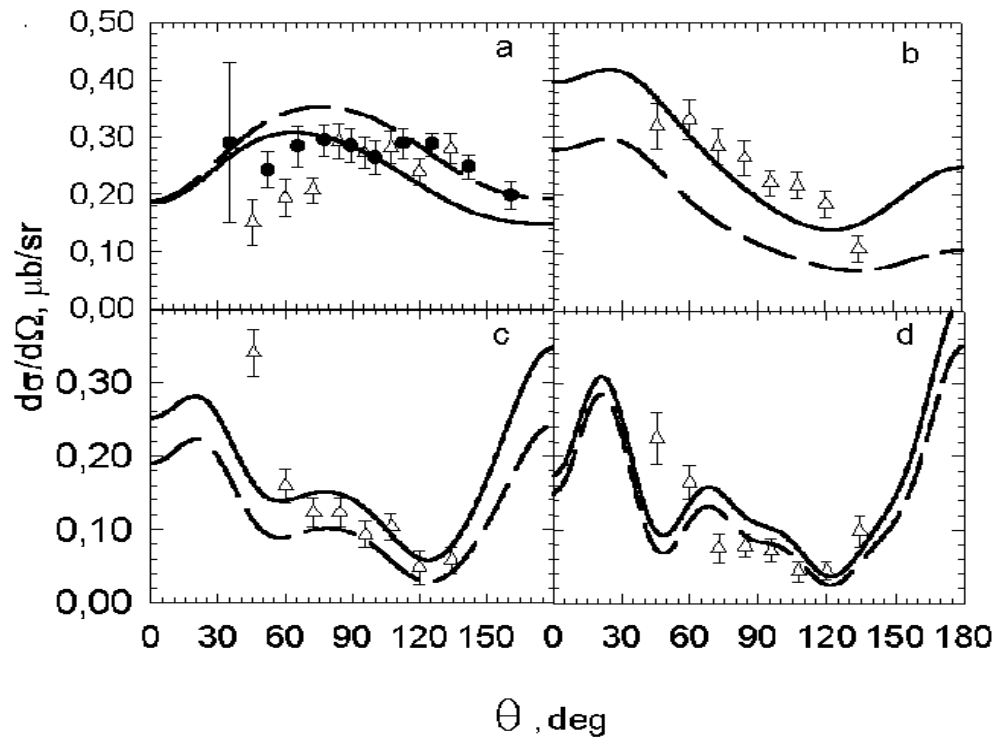

Figure 6: Differential cross section $d \sigma / d \Omega$ for $\gamma p \rightarrow \eta p$ calculated in the c.m. system for the lab photon energies $K_{0}=1025 \mathrm{MeV}$ (a), $1325 \mathrm{MeV}$ (b), $1625 \mathrm{MeV}$ (c), and $1925 \mathrm{MeV}$ (d). The dashed and solid curves are obtained within the models including eleven and twelve resonances, respectively. The data are from [15] (circles) and [22] (triangles). 


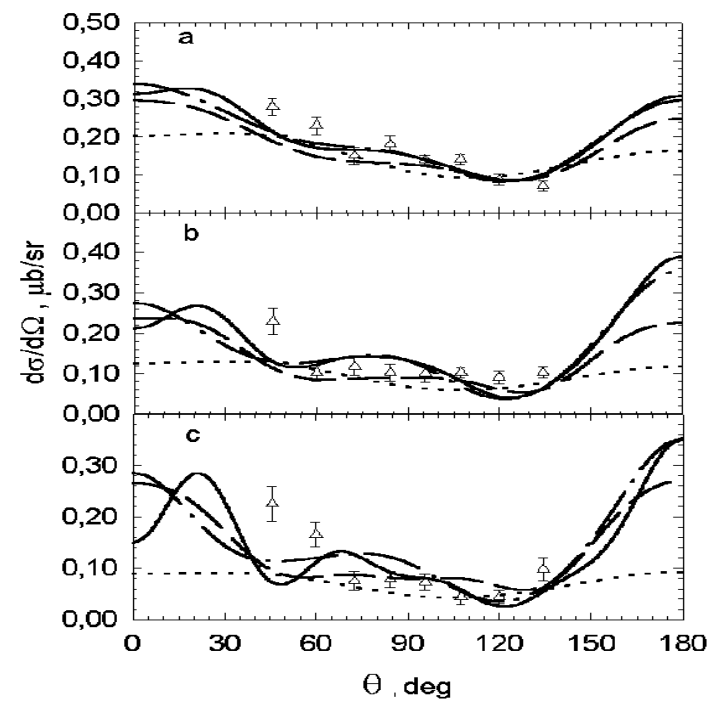

Figure 7: Differential cross section $d \sigma / d \Omega$ for $\gamma p \rightarrow \eta p$ calculated in the c.m. system for the lab photon energies $K_{0}=1525 \mathrm{MeV}$ (a), $1725 \mathrm{MeV}$ (b), $1925 \mathrm{MeV}$ (c). The solid curve is obtained within the models including twelve resonances. The dashed and dash-dotted curves are obtained within the models including ten resonances without $F_{17}(1990), H_{19}(2220)$ and without $G_{17}(2190), H_{19}(2220)$ respectively. The dotted curve is obtained within the models including eight resonances (without $F_{17}(1990), G_{17}(2190), G_{19}(2250), H_{19}(2220)$ ). Data are from 22 . 


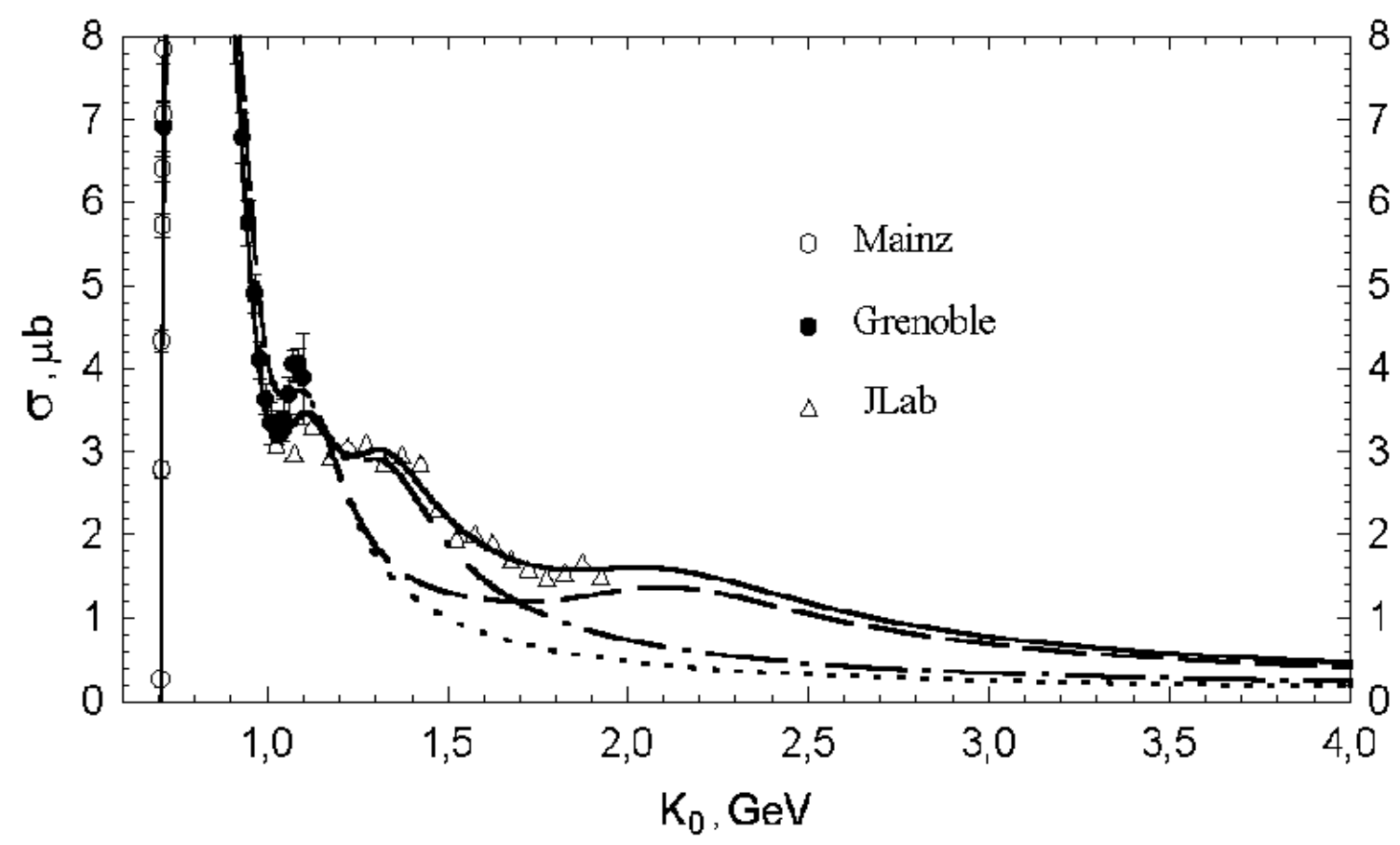

Figure 8: Integrated cross section for $\gamma p \rightarrow \eta p$ as a function of the lab photon energy $K_{0}$. The calculation was performed using the model with twelve resonances (solid curve), with eleven resonances (without $S_{11}(1825)$ ) (dashed curve), with eight resonances (without $F_{17}(1990), G_{17}(2190), G_{19}(2250)$ ), $H_{19}(2220)$ (dash-dotted curve), and with seven resonances (without $\left.S_{11}(1825), F_{17}(1990), G_{17}(2190), G_{19}(2250)\right), H_{19}(2220)$ (dotted curve). The background was treated as in the "hybrid" model of Ref. 21]. The data are from 14, 15, 22]. 